\title{
A survey on the use-cases and deployment efforts toward converged internet of things (IoT) and vehicle-to-everything (V2X) environments
}

\author{
Hamdan Hejazi ${ }^{1, *}$, László Bokor ${ }^{1}$ \\ ${ }^{1}$ Budapest University of Technology and Economics, Department of Networked Systems and Services \\ Magyar tudósok körútja 2, 1117 Budapest, Hungary \\ *e-mail: hhejazi@hit.bme.hu
}

Submitted: 19/05/2021 Accepted: 01/10/2021 Published online: 27/12/2021

\begin{abstract}
In the past few years, automotive Internet of Things (IoT) solutions have become one of the most significant IoT application areas in the shape of vehicular communication to connect vehicles and such the so-called Internet of Vehicles (IoV) to be used in Intelligent Transportation Systems (ITS) environments. With an increasing level of cooperation, ITS could facilitate smart city operations by providing cooperative intelligent traffic solutions. Modern Cooperative ITS (C-ITS) solutions have started to be implemented in the whole world with various deployment models and significant improvements in the integration of Vehicle-to-Everything (V2X) communication and IoT solutions. To highlight the current V2X technology evolution towards an IoT/IoV era, this paper presents a comprehensive survey about the convergence between IoT and V2X use-cases together with their supporting technologies in the cooperative ITS ecosystem worldwide. We show how IoT could enable advanced V2X applications to get widespread and increase ITS efficiency.
\end{abstract}

Keywords: IoT-V2X convergence; Internet of Vehicles; Cooperative ITS; technology evolution; V2X applications

\section{INTRODUCTION}

Internet of Things (IoT) enables an enormous number of devices around the world to have immediate access to information, communicate and transfer data gathered from different environments to the upper layer of IoT platforms, promptly to the applications, or to the cloud for computing and data processing to provide a valuable service. According to Cisco, 25 - 50 billion 'things' will be connected to the Internet by 2020 [1]. IoT can be presented as a network of surrounding things connected to the Internet, such as various vehicles that can be monitored, detected, or controlled. The cars are embedded with sensors to sense the environment and communicate with other vehicles and ITS stations. For that, the environment is monitored by intelligent sensing and measurement techniques, and the collected data is transferred to the cloud by communication procedures between the widest scale of interconnected devices ("things").

IoT has been adapted to incorporate the digital information and the real world of devices; however, IoT platforms and management tools need to provide solutions to meet connectivity, heterogeneity, security, scalability, and data handling. The fastgrowing IoT paradigm trends adumbrate that IoT will gain higher importance in several industries in the coming years [2]. This aggressive growth makes the emerging automotive industry and vehicular communications pose one of the most challenging tasks in integrating IoT and V2X.

V2X refers to the technology family that enables cars or any vehicles and even vulnerable road users (VRUs) to communicate and send messages between them and the surrounding environment [3]. This connectivity will enhance the traffic flow, navigation, and collision avoidance and provide an unobstructed view of traffic situations in the most hazardous situations. V2X enables vehicles to share dynamic data and information (such as speed, position, heading, curving, loss of stability, braking, etc.) in real-time to provide safer transportation and to help various value-added services like advanced traffic management.

The global relevance of IoT and how it can be applied to several domains (such as vehicular communications using a plethora of things like sensors, actuators, and devices) to communicate with each other directly or indirectly via the Internet 
introduce significant challenges in how IoT can be involved in vehicles industry. With these advantages of V2X, Cooperative Intelligent Transportation Systems (C-ITS) can be built with many valuable services [4].

It is expected that in a time frame till 2025, 150B USD will be generated as revenue for connected vehicles with a number near to $100 \mathrm{M}$ cars around the world [5][6]. The expected volume of data transmitted between the cloud and vehicles will be around 100 petabytes per month [7][8]. Also, there will be many deployment examples of ITS's worldwide with valuable automotive services provided to future connected vehicles, which will enable safety during the driving, the efficiency of the energy consumption, and reduce the emissions from the cars.

Cooperative Intelligent Transportation Systems incorporate vehicles that are interconnected with each other and various elements of the road infrastructure and the overall transportation ecosystem. ITS aims to fewer accidents, less congestion, and reduced emissions. The application of V2X communication technologies in different contexts such as Vehicle-to-Vehicle (V2V), Vehicleto-Network (V2N), Vehicle-to-Pedestrians (V2P), Vehicle-to-Infrastructure (V2I), Vehicle-to-Grid (V2G), and Vehicle-to-Device (V2D), could create the essential infrastructure to help to reach this vision [9]. V2X augments sensors, computer vision, and live data analysis with different information sources, which can be supported by integrating IoT and V2X technologies and allow vehicles to see intersections and create/extend the visual horizon. Vehicles can connect and communicate in different connectivity options, such as the currently available Wi-Fi-based ITS-G5/DSRC without any pre-deployed communications equipment in the middle or by mobile cellular solutions such as 4G LTE technology. Cellular V2X (C-V2X) is also coming into the picture: LTE and 5G NR C-V2X are about to be deployed soon [10].

This paper introduces the convergence of IoT and V2X and its relation to enable ITS to accomplish and provide a valuable service by using most modern technologies such as 5G technology. In section II, we introduce the meaning and explain the concept of the Internet of Vehicles (IoV). In section III, joint usecases for using IoT with V2X/C-V2X are presented. Then, in section IV, V2V with IoT communication technologies will be illustrated, such as the 5G technology convergence of Cellular IoT for V2X. After that, Different deployment models/examples of intelligent transportation systems will be given our survey results about these deployment activities besides the advantages of adding IoT features to them in section V. The study ends with concluding our key findings in section VI.

\section{THE CONCEPT OF THE INTERNET OF VEHICLES (IOV)}

The Internet of Vehicle (IoV) is a platform consisting of connected vehicles with V2X communication systems enhanced by IoT's power and allowing transferring and exchanging information in real-time with different relevant actors and surrounding environments with high reliability (Fig.1). These actors could be vehicles, infrastructure, pedestrians, communication network devices. IoV will help solve the common problems related to traffic, such as safety and congestion and reduce traffic crashes and emissions to save the environment [11].

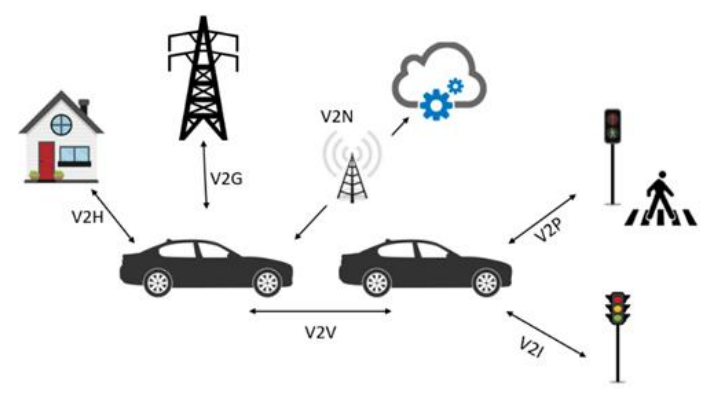

Figure 1. Internet of Vehicles (IoV)

An IoV platform can be created by starting with implementing a reliable V2X communication system. Then, the next phase is to integrate different devices and sensors to measure and monitor the vehicles for sensing the environmental conditions, getting information, and collecting data. This data can be analyzed locally by a middleware component place closer to the end-users according to the Mobile Edge Computing (MEC) paradigm. Another choice is sending the gathered data to the upper segments, known as cloud computing, to analyze the data and achieve the goal of integrating vehicular networks, also using different types of communications [12].

The first wave of solutions for initiating such systems will be based on the IEEE 802.11p technology. After Release 14 and beyond, cellular solutions based on sidelink communications will be implemented, C-V2X could also gain momentum. After detected information processing, representation, and visualization in deployment models such as ITS, the analyzed data dispatches towards end-users as apps on the mobile applications or as information to other vehicles to inform them about the traffic situations and detect the expected hazards [13].

However, IoV has many critical challenges concerning the integration of connected vehicles with IoT. Firstly, (i) heterogeneous IoT devices and cars pose a serious problem, which requires extensive connectivity to billions of heterogeneous IoT devices such as sensors, actuators with various 
vehicles from different manufacturers. Secondly, (ii) communications systems with network and low power communication protocols are required with stable wireless links to maximize the throughput for increasing transmission intensity of data exchange, driver safety, and awareness of critical situations [11]. The 5th generation of mobile cellular architectures becomes relevant to tackle the beforementioned challenges, creating a flexible structure and advanced communication techniques designed to adapt to various use cases in IoT. Thirdly, (iii) security is also a considerable challenge. Security breaches in the vehicular domain could easily result in unacceptable consequences such as financial losses and loss of credibility. Alerting services such as self-driving mode charging, traffic rerouting, and localization and security procedures in case of abnormal status will enhance the security in different use cases of IoT integration with V2X. Fourthly, (iv) data management accounted as a significant challenge because of including data collection and data analytic, which need to handle promptly and actuate data providing in real-time and support data visualizations, management, incorporation, and security [14].

\section{JOINT USE-CASES OF IOT AND V2X/C- V2X}

Vehicular communication systems are nowadays limited to vehicles and roadside infrastructure nodes; they can be connected directly or indirectly to various fields and environments such as traffic management centers, pedestrians, devices, networks, or even the power grid. Implementation of IoT with these scenarios will provide more privileges and a clear vision of situations to add value and build intelligent life and enhance connected vehicles with IoT. In the below subsections, we group and introduce the identified join use-cases of IoT and V2X/C-V2X (Table 1).

\section{Vehicle-to Vehicle with IoT}

Different types of vehicles, such as autonomous vehicles or smart ones, can communicate with each other directly by ad hoc networking techniques or indirectly by relying on the infrastructure using different protocols and networking topologies. The original goal of $\mathrm{V} 2 \mathrm{~V}$ systems was to alert drivers about abnormal events but not to control the vehicle. Later, with the advancement of self-driving vehicles, ADAS (Advanced Driver-Assistance Systems) solutions improved to act instead of the human drivers, such as steering around the obstacles or breaking [15]. For instance, if there are two vehicles with $\mathrm{V} 2 \mathrm{~V}$ following closely each other, and the vehicle in the front performs emergency braking, the following vehicle will receive a warning such as a flashing red light about that event. It could take action immediately by braking or overtaking. Such cooperative operations do not affect traffic. Simultaneously, the other vehicles with V2V systems and driving nearby will also get the notification that the drivers in the neighborhood can use to save time and be part of a more efficient traffic environment.

There are so many different use cases about how connected vehicles will enhance traffic efficiency and safety. These include alerting services such as the approach of emergency vehicles and traffic jams to enable collision avoidance in addition to entering intersection warnings, leaving highways, pre-crash indication, lane changing unexpected stop warnings, and overtaking between two vehicles [16]. IoT can be involved in all previous use cases by assisting the vehicles and drivers to be proactive because of collecting various data and information from different sources such as vehicles or infrastructure represented by roadside units. Then, alert the drivers or cars about the traffic, whether it is crowded or not, help drivers and cars make appropriate decisions and improve traffic efficiency.

\section{Vehicle-to-Infrastructure with IoT}

Communication of vehicles with traffic signals and roadside units or other stationary devices are known as Vehicle-to-Infrastructure (V2I). The cars and other road users send the collected data to one of the nodes previously presented as the contact point of the infrastructure and vice versa. Infrastructure components, including traffic lights, lane markings, and road signs, are also able to provide information to vehicles. The V2I context is the closest one to IoT because it groups the cars or vehicles (V) in general with other things ( $\mathrm{T}$ ) to give the meaning of Vehicleto-Things (V2T) with various missions such as changing traffic lights or alerting drivers about road hazards, including obstacle discovery, accidents reporting, closed roads, roadworks or maintenance on the way and different hazardous location warnings [17].

IoT can be implemented in V2I use-cases to enable valuable advantages. For instance, IoT will provide more detailed information about the surrounding environment and traffic conditions such as weather conditions, roadside construction, faulty traffic lights, or even accident services such as Internet access. As a use-case of IoT applications with V2I, IoT technology could provide intelligence services such as automatic parking payments, toll charges, and creating dynamic traffic light control to manage the traffic lights for approaching emergency vehicles such as a fire brigade or police cars. Moreover, the information coming from IoT platforms can provide valuable information about the recommended speeds, sharp curves warnings with ideal speed to prevent accidents, slippery roads, and environment temperature [18]. 


\section{Vehicle-to-Pedestrian with IoT}

According to The U.S. Department of Transportation (USDOT), in 2013, there were 32,719 people killed in motor vehicle traffic crashes and incidents in the United States. Pedestrians and other road users' occupants made up 17 percent of these victims [19]. Moreover, according to a global status report on road safety 2018 by the World Health Organization, the number of annual road traffic deaths has reached 1.35 million. Road traffic injuries are currently the leading cause of death for pedestrians, cyclists, motorcyclists, and young adults aged 5-29 years [20]. The number of roadway fatalities has a steady reduction in recent years because of enabling different safety approaches. This effect is also expected with the spreading of vehicular communications such as V2V, V2I, and Vehicle-to-Pedestrian (V2P). The V2P approach includes vulnerable road users known as pedestrians (such as people walking, disabled people with wheelchairs or other mobility devices, passengers who come in and out from buses and trains, and also cyclists) into the V2X ecosystem.

$\mathrm{V} 2 \mathrm{P}$ enhances the pedestrian's safety by using personal mobile devices or wearables to receive and send notices about the horizon by sending a note from a mobile app to the cloud in a related IoT platform [21]. Regarding that, drivers and vehicles in the area will receive alerts about that on concise notice. For instance, when pedestrians perform illegal crossing because of blind disability or low vision to the traffic signal, an automated call from a pedestrian's smartphone alert both the driver and pedestrian to turn on or stop. IoT can be involved in this use case by providing information collected by intelligence cameras or sensing the object crossing the crosswalk during the red signal and then alerting the driver about the illegal crossing. Moreover, it can use in the safety of public zones, such as the drivers on the way of the schools will receive warnings when students go out and make places crowded places of them.

\section{Vehicle-to-Network with IoT}

Mobile networking is an essential technology for vehicular communication. Vehicles can communicate with each other or with other environment objects wirelessly and bidirectionally by two distinct approaches: infrastructure-based and ad hoc networks. The infrastructure-based system depends on the cellular concept and terrestrial network infrastructure support. Ad hoc networks become available because of the widespread availability of wireless communications with no need for self-organizing networks and not require a pre-established infrastructure such as Mobile Ad Hoc Networks (MANET) [22]. MANET is a combination of wireless mobile nodes that exchange information and build networks dynamically without employ any pre-existing fixed network infrastructure or a centralized administration. The Vehicular version is known as Vehicular Ad Hoc Network, a variation of MANETs with nodes of vehicles [23].

IoT is mainly a network that extends the Internet, relying on various communication solutions between the IoT module and the platform such as LoRaWAN, NB-IoT, and 5G cellular [24]. These technologies are already implemented in vehicular communications by using the communication in the network layer and identification layer of IoT platforms and can also be used to enhance traffic efficiency. This integration between V2N and IoT with various supported communication protocols enables services in different communications scenarios in case of partial unavailability of services such as Internet accesses and cloud computing [25].

\section{Vehicle-to-Home and Vehicle-to-Grid with IoT}

In the case of Vehicle-to-Home (V2H) scenarios, vehicles are exchanging information with a smart home environment for advanced control and efficiency. Vehicle-to-Grid (V2G) communication is a power grid extension system that regulates the vehicle's charging rate or returns electricity to the

Table 1. Use-cases of V2X/C-V2X and possible IoT enhancements

\begin{tabular}{|c|c|c|}
\hline Use-cases & $\begin{array}{l}\text { V2X Communication } \\
\text { context }\end{array}$ & IoT enhancement \\
\hline Proactivity & Vehicle-to-Vehicle (V2V) & $\begin{array}{l}\text { Awareness by collecting data and information from } \\
\text { different resources to make the best decision. }\end{array}$ \\
\hline Traffic Efficiency & $\begin{array}{l}\text { Vehicle-to-Infrastructure } \\
\text { (V2I) }\end{array}$ & $\begin{array}{l}\text { Comprehensive behavior by collecting data and } \\
\text { information from the surrounded environment. }\end{array}$ \\
\hline Safety of Pedestrian & $\begin{array}{l}\text { Vehicle-to-Pedestrian } \\
\text { (V2P) }\end{array}$ & $\begin{array}{l}\text { Personal mobile devices to receive notices about the } \\
\text { horizon. }\end{array}$ \\
\hline Connectivity & $\begin{array}{l}\text { Vehicle-to-Network } \\
\text { (V2N) }\end{array}$ & $\begin{array}{l}\text { Connectivity is provided by a variety of } \\
\text { communication solutions, technologies, and platforms. }\end{array}$ \\
\hline Home Management & Vehicle -to-Home (V2H) & $\begin{array}{l}\text { Support Recourse and smart home applications } \\
\text { control. }\end{array}$ \\
\hline Renewable Energy & Vehicle-to-Grid (V2G) & $\begin{array}{l}\text { Renewable power storage alternative: improve power } \\
\text { consumption. }\end{array}$ \\
\hline
\end{tabular}


grid. V2G could provide an energy storage alternative such as electric vehicle batteries could be used as alternatives for energy storage to provide home devices with the power needed. IoT in $\mathrm{V} 2 \mathrm{H}$ can provide innovative home applications concerning vehicles such as air condition to control the home's temperature during driving and before reaching the house. In V2G, IoT platforms used in the power grid for intelligence charging determine the best time to charge vehicles batteries and improve power consumption and safety system [26].

The use-cases of interactions between IoT and vehicular communication will introduce and invent so many domains and applications to add value in different fields of life. A good example is the EC H2020 AUTOPILOT project, which also provides a vision for Vehicle-to-Users (V2U), Vehicle-toOwner (V2O), and Vehicle-to-Maintenance (V2M) [27].

\section{TECHNOLOGIES SUPPORTING THE CONVERGENCE OF IOT AND V2X COMMUNICATION}

Different types of V2X standards deal with vehicular networks in various layers. The IEEE 802.11p Wireless Access in Vehicular Environment (WAVE) standardization which is related to all layers and processes, originates from the allocation of the Dedicated Short-Range Communications (DSRC) spectrum band to use for safety applications in the U.S. [28]. Its European proportionate is ITSG5, still relying on Wi-Fi-based technology. In recent years new cellular V2X (C-V2X) standard has been established and defined basic functionality by the 3rd Generation Partnership Project (3GPP), which provides vehicular communication standards for both Long Term Evolution (LTE) and 5th generation (5G) New Radio (NR) releases. Their goals are to enable extended reliability and improved range for enhanced safety and efficiency of the traffic, which can be exploited with the 5G NR technologies from 3GPP Rel 16 onwards [29][30]. Moreover, in the 3GPP specifications Rel 12, ProSe (Proximity Services), a device to device (D2D) technology, is introduced to enable LTE devices to communicate and detect each other directly depending on the various enhancements added to existing LTE standards, including new features such the "sideline" type of link for direct communication between devices. ProSe-enabled devices will be utilized in the mission-critical communications sector, such as V2X public safety applications, especially in $\mathrm{V} 2 \mathrm{P}$, such as traffic relaying for wearables [31].

IoT solutions use various communication solutions between the IoT module and the platform, such as LTE-M, LoRaWAN, NB-IoT, ZigBee, ZWave, Bluetooth / BLE, Sigfox, NFC, 6LowPAN,
IEEE 802.11af (White-Fi), IEEE 802.11ah (HaLow), 2G/3G/4G/5G cellular [12][32]. These technologies are already implemented in vehicular communications by using the communication and identification layer of IoT platforms, which can also enhance traffic efficiency by enabling services with other communication scenarios in case of partial unavailability of services such as Internet accesses and cloud computing.

The power of the 5th generation $(5 \mathrm{G})$ cellular network connectivity with the broadest possible applications can be considered the gateway to the ubiquitous vehicular network access and V2X scenarios integration. This will enable vehicles to access any remote resources using coverages of $5 \mathrm{G}$ supported architectures to be connected to new radio technologies with ultra-low latency and high reliability [33]. In addition to that, $5 \mathrm{G}$ will provide a unified communications platform for the IoT, which extends the mobile communication services for heterogeneous devices offering the $5 \mathrm{G}$ ecosystem to be integrated with C-V2X and support Internet-ofVehicle (IoV) use-cases [34].

There is always a question about how the fifth generation of mobile cellular technology will involve our life in the future, especially in V2X and IoT. Regarding the Internet of Things, 5G will provide enough bandwidth to include an enormous number of devices that can communicate with each other. Still, this ability is not a new feature compared to IoT, but the unique privilege is to connect almost unlimited devices on the same $5 \mathrm{G}$ network. The combination and development of IoT and $5 \mathrm{G}$ automatically leads to the IoV era by providing very low latency within $5 \mathrm{G}$ NR vehicular communications, robust traffic management, and collision avoidance probabilities [35].

The automotive industry keeps tracking the new techniques introduced for future V2X by cooperating with the 3GPP and enhancements provided on parts of $5 \mathrm{G}$ releases. This evolution requires $\mathrm{V} 2 \mathrm{X}$ upgraded systems and technical designs except for native support by the $5 \mathrm{G}$ network to create valuable services and application scenarios such as vehicle platooning, dynamic ride sharing, remote driving, and collaborative environment perception. Implementing $5 \mathrm{G}$ with different V2X layers will introduce the next generation of network systems with several challenges, such as control signaling among vehicles and interference management [36].

These advantages provided by 5G [37] can reduce the latency to near zero and use the most critical digital payloads with high security. In addition to that, $5 \mathrm{G}$ can give us accurate in-time solutions in mobility and connected devices, especially in V2X such as 5G smart roads, to recognize critical indicators of an accident and trigger actions to alert the connected vehicles. Also, 5G provides additional 
privileges in the $\mathrm{V} 2 \mathrm{X}$ industry, such as continuous coverage along tracks in high-speed trains and highways, enabling reliability and availability with low latency under high speed with full utilization traveling 500 kilometers per hour. 5G can give imagination about the future of $\mathrm{V} 2 \mathrm{X}$ where autonomous vehicles do not require stoplights. The infrastructure provides self-reports such as potholes in the road, connected smart stop and street lights and intelligent parking in the urban, and proactive notification about services needed for vehicles and sent to the driver for scheduling a time in the calendar smartphone maintenance service time [38].

Vehicle manufacturers and automakers are planning and racing for developing future electric and autonomous vehicles. E.g., Ford and Mercedes are implementing $5 \mathrm{G}$ and IoT health technology by using in-seat sensors and infrared cameras to enable built-in health monitoring systems which can measure body temperature and monitor vital signs for drivers and passengers as well such as blood pressure and heart rate on the dashboard of the vehicle. Then they send the collected data by using $5 \mathrm{G}$ technology to the cloud for analysis, which could help be proactive and prevent unexpected incidents such as heart attacks by alerting the driver about any abnormal health events that could be occurred during the driving. The system could even stop the vehicle if required. Also, it is able to inform the emergency directly about the situation and the location, followed by the transmission of the vital data to a medical center to provide a clear vision of the driver or passenger's health [39] [40].

\section{IOT AND V2X CONVERGENCE IN RECENT C-ITS PROJECTS}

In the last few years, governments worldwide have implemented significant improvements in the ITS/CITS domain. Vehicle manufacturers were also able to introduce communication techniques to improve roadway safety and effectively manage traffic flow in the longer run. These active deployments allocated in different geographical areas or regions involve two different technologies: C-ITS (Europe ITS-G5) and DSRC (U.S. - WAVE) based on IEEE $802.11 \mathrm{p}$ protocol. The European Cooperative Intelligent Transport Systems (C-ITS) activities are continuously sharing the experience and knowledge about the implementation of these modes between member states. The U.S. Department of Transportation (USDOT), which is a federal Cabinet department of the U.S. government concerned with transportation, also manages several ITS projects and publishes results periodically [41]. These deployment activities also rely on IoT platforms or parts of them, such as cloud or edge computing or data management. In the following sections, we will introduce recent C-ITS/V2X deployment projects in different areas worldwide with their implementations, expectations, and future enhancements that will apply to these projects in a converged IoT/V2X ecosystem (Table 2).

\section{CROCODILE}

The CROCODILE corridor was conceived in 2013 and comprised road network elements of 13 Central and Eastern European countries with different network characteristics and crosses bi-national borders at more than 15 locations to increase road safety and efficiency along the corridor motorways [42]. Public authorities, road administrations, and traffic information service providers from different member countries cooperate to make the infrastructure more efficient in data and information exchange between public and private stakeholders. The primary goal is to provide information services in the whole corridor for the end-users and crossborder travelers. The core activities of CROCODILE are represented in data collection, processing, access, and end-user services. The CROCODILE platform had challenges with the gaps in traffic monitoring and management, which introduced to CROCODILE 2 to include traffic and road data exchange, implementation of National Access Points, which have been established by the delegated regulations between member states, cross-border agreements, and traffic management plans. The CROCODILE solution depends on the infrastructure and can be used by drivers and pedestrians through highway applications and crossing between the borders along the corridor. The IoT implementation related to the project is presented in the deployment of equipment to provide data collection (such as CCTV and sensors) and traffic management centers for improving infrastructure for data processing which is one of the focus topics in the CROCODILE 2 project [43].

\section{C-ROADS}

C-ROADS is a C-ITS platform co-funded through the 2015 call of the Connecting Europe Facility (CEF) and implemented in 43 European cities, 16 European countries' core members, and associate members Australia and Russia [44]. The deployment model of C-ROADS consists of group C-ITS Actors represented as organizations or entities. The mission is to operate C-ITS stations and/or provide C-ITS services through the information-sharing network using AMQP (Advanced Message Queuing Protocol) broker(s). In addition, they plan to use Hybrid C-ITS, which enables multiple communication channels for transmission of C-ITS messages with conditions of the policy, location and requirements set [45].

The purpose of the model is to create cooperation on all deployment levels of C-ITS and different stakeholders. Because of various country regulations and incoherence in the implementation issues, the 
member states should detect and address these challenges and evaluate them to reach interoperable C-ITS services in all C-Roads member states [46]. $\mathrm{C}$-roads is built to send and receive messages between vehicles $(\mathrm{V} 2 \mathrm{~V})$ or between vehicles and infrastructure (V2I) with various services known as "Day-1-services" encompassing messages about hazardous location notification, signage application, road-works warning (RWW), and infrastructurerelated data [47]. The noticeable challenge in CROADS is implementing the platform in different countries could be led to interference, such as ITSG5 frequency should not interfere with older systems and regulations in member states and implement and support other vehicular communications use cases such as V2P. C-ROADS implemented complete IoT platforms as parts of the project's system architecture, starting with on-board units (OBU), representing the sensors in vehicles, and Road site units (RSU) which sent/receive data such as C-ITS messages. After that, the collected data transfer to central systems for processing and distribution due to the Integration Platform (IP), responsible for collecting C-ITS messages from each back office and forwarding these messages to the other associated members [48].

\section{NordicWay}

NordicWay was a three-year (2015-2017) real-life deployment pilot established to test cellular C-ITS services' interoperability in the whole NordicWay network, including 4 Nordic countries: Denmark, Finland, Norway, and Sweden [49]. NordicWay provides services for both passenger and freight traffic. In the NordicWay environment, vehicles indirectly communicate via cellular communications as the underlying communication infrastructure. NordicWay was followed by broader deployment in the Nordic countries and Europe (NordicWay2 and C-ROADS Platform).

NordicWay provided "Day-1-services" such as hazard warning services through cellular networks. After that, NordicWay2 was established to offer interoperable Day-1 and Day 1.5 C-ITS services and support infrastructure readiness for connected and automated driving such as enhance traffic safety, support cloud to cloud hybrid communication, and contribute to European CCAM (Cooperative, Connected and Automated Mobility) harmonization through C-ROADS. Last year, NordicWay 3 - Urban Connection was signed by the partners and the E.U. in Eindhoven in June 2019. One of the objectives for pilot deployment in NordicWay and NordicWay2 projects is an IoT platform implementation for support infrastructure readiness for connected and automated driving and scaling up C-ITS services by supporting cloud-to-cloud hybrid communication from the upper layer of this IoT platform [50].

\section{Scoop@F}

SCOOP is a project for C-ITS pilot deployment launched in (2014-2019) in France to connect 3,000 vehicles with $2,000 \mathrm{~km}$ of road network with various typologies to improve road safety and efficiency. The first part (2014-2015) was related to ITS-G5 and priority services such as Day-1 C-ITS services. The second part (2016-2019) was about hybrid cellular/ITS G5 and additional services, such as enabling large-scale deployment and contributing to C-ITS interoperability in the E.U. [51]. The SCOOP@F system has been developed with vehicle manufacturers to build a robust backend architecture, including a traffic management system to enable automated driving and react to road conditions adaptively. ITS central station contains SCOOP IoT platform connecting to the roadside unit infrastructure, road operator On-Board Unit (OBU), or User On-Board Unit [52]. The closing of the SCOOP project took place on November 20th-21st, 2019 , in Bordeaux. The next step for the project is testing the interoperability among different systems by using the SCOOP@F system with other countries and existing C-ITS implementations [53].

\section{SAFER -LC}

SAFER-LC (Safer level crossing by integrating and optimizing road-rail infrastructure management and design) is an E.U. project started on May 1, 2017, with durations three years composed of 17 partners from 10 different countries represented in railway infrastructure owners, road and railway operators, academic research centers, universities, and industry suppliers. SAFER-LC provides safety monitoring systems and advanced mobile communication technologies, including the use of IoT platforms parts such as CCTV and cooperative communications to develop innovative solutions [54]. The main objectives of SAFER-LC, including firstly, developing innovative solutions to enhance the safety of level crossing for road and railway users. After that, demonstrate new technologies and solutions that can be integrated and provide the required performance and manage the level crossing the user's perspective and behavior. Finally, deliver these solutions for implementation and develop a toolbox with a user-friendly interface to help both rail and road managers to improve the safety at level crossings [55].

\section{AUTOPILOT}

The AUTOPILOT (AUTOmated driving Progressed by Internet Of Things) project is a pilot of the IoT European Large-Scale Pilots (LSP) Programme. LSPs Programme launched in 2016 to apply IoT approaches and deployment of IoT solutions through the integration of advanced IoT technologies. The LSPS includes seven pilots 
dependent on addressing the IoT applications based on European relevance.

These pilots are: (1) ACTIVAGE for breaking barriers for sustainability Active and Healthy Ageing through IoT technologies, (2) IOF2020 for IoT deployment with strengthening the competitiveness of farming and food chains in Europe, (3) MONICA for sound and security solutions for large open-air events in the smart city, (4) SYNCHRONICITY for creating single digital city market of Europe, (5) U4IoT for actively engage end-users and citizens to achieve IoT societal acceptance, (6) CREATE-IoT to stimulate collaboration between IoT initiatives, by supporting the development and growth of IoT ecosystems based on available technologies and platforms, and finally, (7) AUTOPILOT to unlock the potential of IoT to participate the next level of autonomous driving [56].

AUTOPILOT was concluded between 2017-2019 with the cooperation of 45 partners from 14 European countries and one from South Korea to increase traffic safety and create new opportunities for mobility services. AUTOPILOT was initiated to expect that automated vehicles will be part of the IoT revolution because IoT connectivity is expected to have essential impacts on highly and fully automated driving generations [57].

AUTOPILOT used IoT-enabled autonomous driving vehicles in a connected real-life testbed environment at large-scale pilot sites in the Netherlands, Italy, France, Finland, Spain, and South Korea. The focus was on safety-critical aspects of automated driving and providing valuable services such as autonomous car sharing, automated parking, and building better digital maps for autonomous vehicles [58].

\section{InterCor}

Interoperable Corridors (InterCor), an innovative European project, was established in 2016 and ended in 2019, co-financed by the European Union under the Connecting Europe Facility (CEF) 2014-2020 had 16 project partners. The project aimed to connect and create Interoperable Corridors linking the C-ITS corridor initiatives of the Netherlands C-ITS Corridor, the French corridor defined in the SCOOP@F project, and extended to the United Kingdom and Belgian C-ITS initiatives. The goal was to enable interoperable cross-border C-ITS services through ITS-G5, cellular, and hybrid communication within the four-member states and tested by several test fests with vehicles from different member states and others driving on each other's test sites [59].

InterCor was established to achieve a sustainable network of corridors providing continuity of C-ITS services and offering a testbed for beyond Day $1 \mathrm{C}$ ITS service development. The project members worked together to develop and test effective new services for road users on highways, such as road works warning, truck parking, green light optimal speed advisory, and multimodal cargo transport optimization. The international cooperation of InterCor relying on the combined technologies within the project proved that safer and more efficient mobility is achievable. For IoT aspects, InterCor used road infrastructure for exchange data and connectivity between the project members, which enable heterogeneous IoT platforms to communicate and achieve the proposed results from the project [60].

\section{DRIVE C2X}

DRIVE Car-to-Everything (C2X) project started in 2011 and finished in 2014. The project was supported by the European Union and had 33 partners and 13 associated partners. The main goals of DRIVE C2X were accelerating cooperative mobility and creating European references for cooperative driving by bringing together seven national test sites in Germany, France, Finland, Italy, Netherlands, Spain, and Sweden for enabling a consistent Europe-wide testing environment [61].

The project aimed at operational field tests using the cooperative driving reference system to enable reliable results under real and various conditions, providing valuable and accurate information about road safety and efficiency and the environmental and economic benefits of cooperative driving for public users and decision-makers. DRIVE C2X succeeded in accomplishing the $\mathrm{C} 2 \mathrm{X}$ system prototyped and evaluated under real-life conditions with Europeanwide interoperability. The project results positively impacted safety, such as In-Vehicle Signage speed limit could reduce, on average, $23 \%$ in fatalities and $13 \%$ in injuries, and weather warning leads to $6 \%$ fewer victims and 5\% fewer injuries. Moreover, the positive impact also appeared on efficiency, environment, and user acceptance. The impact of invehicle signage grows with penetration rate, and green light optimal speed advisory reduced the fuel consumption and $\mathrm{CO} 2$, and 9 out of 10 test users liked the cooperative system. They wanted to use it if it were available on their vehicles [62]. The IoT aspects are not so apparent in Drive C2X. However, one of the project's primary goals was to collect massive data from various resources and help the drivers making the best decision. This mission will not be successful without involving IoT privileges.

\section{Other European C-ITS activities}

Other deployments or activities aimed to enable road safety, efficiency, cooperative services, and save people's lives. For instance, Cooperative, Connected, and Automated Mobility (CCAM) is a platform to enable the C-ITS in the European union by testing and exchange experiences from current 
pilots and discovering the issues related to achieve full convergence of all developments [63].

European Corridor - Austrian Testbed for Cooperative Systems (ECo-AT) is an Austrian project established to create ITS applications with partners Netherlands and Germany to build cooperation between industry manufacturers and road operators for C-ITS services deployment. The project ECo-AT had two phases: the first phase for cooperative services tested and in the second phase: these services and systems have been implemented [64].

Moreover, there are several other deployments, projects, or groups established for a long time to achieve and build successive automotive innovations. E.g., PROgraMme for a European Traffic of Highest Efficiency and Unprecedented Safety (PROMETHEUS) from 1986 to 1994 to build the basis of cooperative ITS and automated driving, or the Safe Intelligent Mobility Test field Germany (simTD) project from 2009 to 2013 for cooperative V2X intelligent communication systems testing and development to achieve road safety and mobility enhancements [65]. Furthermore, Amsterdam Group to achieve and improve road safety and mobility depending on European-wide deployment of C-ITS based on cooperative V2V and V2I communications.

There are still unlimited deployments globally, especially in Europe, to improve and support C-ITS applications and services such as European Standardization Mandate $\mathrm{M} / 453$ to support the Interoperability of Cooperative Systems for Intelligent Transport in the European Community [66]. For IoT aspects in relation with these various projects from the past until now, we noticed that IoT appears either in part or the whole project and helps to achieve the proposed goals more accurately by using IoT platforms with the advantage of connectivity or by using IoT devices which are builtin infrastructures and foundations of these projects and most significant key enablers for them.

\section{USDOT C-ITS Deployment Projects}

USDOT deployment approaches in NYC, Tampa, and Wyoming use Traffic Management Center with IoT management tools to interact with the traffic control. The collected data will be processed and normalized before transmission to the USDOT for additional evaluation. The differences between these approaches could be addressed in the focused objectives and applications or services provided, such as NYC only has Stationary Vehicle Ahead (SVA) application. In contrast, Tampa (THEA) focuses more on traffic monitoring and optimization than Wyoming, which focuses more on Situational Awareness, such as weather warnings [67].

\section{A. NYCDOT Connected Vehicle Pilot}

The NYCDOT is led by the New York Department of Transportation, which began in 2015 and aimed to enhance pedestrians' and drivers' safety by deploying Connected Vehicle (CV) technologies. NYCDOT's objective is reaching Vision Zero goals, which mainly focused on safety applications that rely on V2P, V2V, and V2I communications. These applications' mission is to provide warnings and alerts to road users, enable them to take action in emergency cases, save pedestrians, or reduce injuries or damages to infrastructure and vehicles. The NYCDOT project pursues to encourage auto manufacturers to ideal opportunity to evaluate and create models outfitting the CV technology and safety applications and invest and use Dedicated Short-Range Communication (DSRC) [68]. The deployment of NYCDOT CV Pilot includes approximately 310 signalized intersections for V2I technology, 8 RSUs along the higher-speed way, and 36 RSUs at other strategic locations throughout the city in addition to 8000 equipped vehicles. For pedestrians, approximately 100 users with personal devices that help them safely cross the street receive alerts from Roadside Equipment (RSE) [69]. The project uses an IoT platform architecture to analyze collected data, signals, and vehicles to store in the Traffic Management Center (TMC), then forward to a data processing center and send information to USDOT for evaluation purposes.

\section{B. Tampa DOT Connected Vehicle Pilot}

Tampa-Hillsborough Expressway Authority (THEA), known as Tampa (THEA) Pilot, is another project for USDOT that began in 2015 to address urban congestion and use connected vehicle technology in Tampa's Downtown. THEA pilot deployed various V2V and V2I applications such as Rush Hour Collision Avoidance, Pedestrian Safety, and Traffic Flow Optimization. The THEA CV Pilot includes approximately 47 RSUs and 1020 equipped vehicles with On-Board Unit (OBU) use DSRC 5.9 $\mathrm{GHz}$ utilized as communication technology in addition to $12 \mathrm{~V} 2 \mathrm{~V}, \mathrm{~V} 2 \mathrm{I}$, and V2P applications [70]. The project integrates IoT and V2I by collecting information from the road environments and transmitting the gathering date to RSUs then for performance measures [71].

\section{Wyoming DOT Connected Vehicle Pilot}

WYDOT CV Pilot was deployed in 2015 in I-80 Corridor in the State of Wyoming in the U.S., a critical freight corridor located between the United States, Canada, and Mexico. The WYDOT CV Pilot's objective is to improve the safety road for commercial vehicles and reduce weather-related incidents. WYDOT deploys DSRC communications-based applications that leverage V2V and V2I connectivity. It uses approximately 75 RSUs, and 400 equipped vehicles with OBUs and 
mobile weather sensors [72]. The usage of IoT in the WYOMING pilot project represented in the deployment of connected vehicles technology on partner vehicles, OBUs, RSUs in terms to transfer collected data to TMC systems [73].

\section{Other Relevant C-ITS Projects Around The World}

The efforts on the development and deployment of C-ITS environments keep going not just in Europe and the USA but also in the majority of the world's other countries as well. Good examples are The Ministry of Land, Infrastructure, and Transport (MOLIT) C-ITS program in South Korea, the C-ITS Program for Japan's highway system, and Austroads in Australia and New Zealand, South Korea, and Japan [74].

Austroads is the strategic plan from 2020 to 2024 that contains transport agencies from Australia, and New Zealand cooperated in providing information, tools, and services for these agencies' members to achieve reliable, efficient, and safe mobility to their customers. Austroads focusing on solving problems related to transportation systems for members and their customers. For members, Austroads works on optimizing the infrastructure and benefits of new technologies, improving the safety of road users and workers, achieving sustainability by reusing materials, reducing emissions, and mitigating the impacts of climate change. For customers, Austroads provide services related to decision making depends on the power of data collected, optimizing transport investment, and understanding the customer's needs [75]. For IoT aspects, Austroads uses IoT platforms and devices represent in vehicle manufacturer information systems, accident information retrieval systems, navigation, and driver assistance devices, Bluetooth and cellular devices, and electronic tags [76].

\section{C-ITS Projects with 5G Technology}

The nature of the convergence phenomena between IoT and V2X technologies enables C-ITS projects implementation to provide valuable services by using most modern technologies such as $5 \mathrm{G}$. A recent deployment effort for $5 \mathrm{G}$ in the relation of IoT and V2X could be given with the results of the 5GCAR project, which is a collaboration of 14 partners such as HUAWEI, ERICSSON, and NOKIA to evaluate different use-cases and perform demonstrations. The 5GCAR project testbed was built on the UTAC-TEQMO track in France in 2019 with a $5 \mathrm{G}$ architecture for vehicular automotive usecases. The system's core features consist of 5G NR sidelink communication, 5G NR positioning, and cellular communication using advanced 5G deployment schemes based on Edge Computing and Network Slicing [77].
5GCAR uses an IoT platform, which includes a maneuver planning system which applied in a lane merge coordination use-case to optimize the merging process for vehicles joining the way by sent information from the central maneuver planning system to connected vehicles, besides, to collect information from roadside cameras about unconnected vehicles to provide real-time video between nearby vehicles via $\mathrm{V} 2 \mathrm{~V}$ communication and facilitate safe overtaking maneuver. The connected cars in 5GCAR, which use Lidar sensor, then send the collecting information, which consists of data estimating the position and the speed of detected vehicles to the server infrastructure known as V2I in the IoT platform. The server predicts vehicles' trajectories in real-time by analyzing this information, estimates the expected collision risk, and warns the affected drivers to safely and comfortably avoid the collision. Another important feature and use case in 5GCAR is about road user protection system that distinguishes and detects the pedestrian crosses the street behind an obstacle and stops the vehicle where the pedestrian is close to it and alert the driver about collision warning before [78].

There are also 5G V2X deployment projects around the world in connection with IoT, such as 5GCroCo [79],5G-DRIVE [80], Wuxi Internet of Vehicle (C-V2X) project was deployed by Huawei on a city-level C-V2X network in Wuxi in China [81]. In the USA, the first C-V2X Connected Car Technology Trials were announced in 2017 in San Diego for demonstrating the potential of C-V2X technologies [82].

\section{Conclusions}

V2X can be efficiently supported by a mesh network of connected nodes using wireless communication systems that could interconnect vehicles, roadside units, road smart traffic signals, pedestrians, cloud platforms, home applications, and smart grid infrastructures with the ability to send, receive and transmit signals between each other.

IoT enables an enormous number of devices to have immediate access to information, communicate and collect data from different environments. The gathering data transfers to the upper layer of IoT platforms, promptly to the applications, or to the cloud for computing and data processing to provide a valuable service. The fast-growing IoT paradigm trends adumbrate that IoT will gain higher importance in several industries in the coming years.

Bringing connected vehicles and intelligent IoT environments together makes the approach known as the Internet of Vehicles (IoV) alive. It creates innovative deployment models that enhance the efficiency of traffic, safe driving and opens the way 
Table 2. Recent deployment and $R \& D$ activities

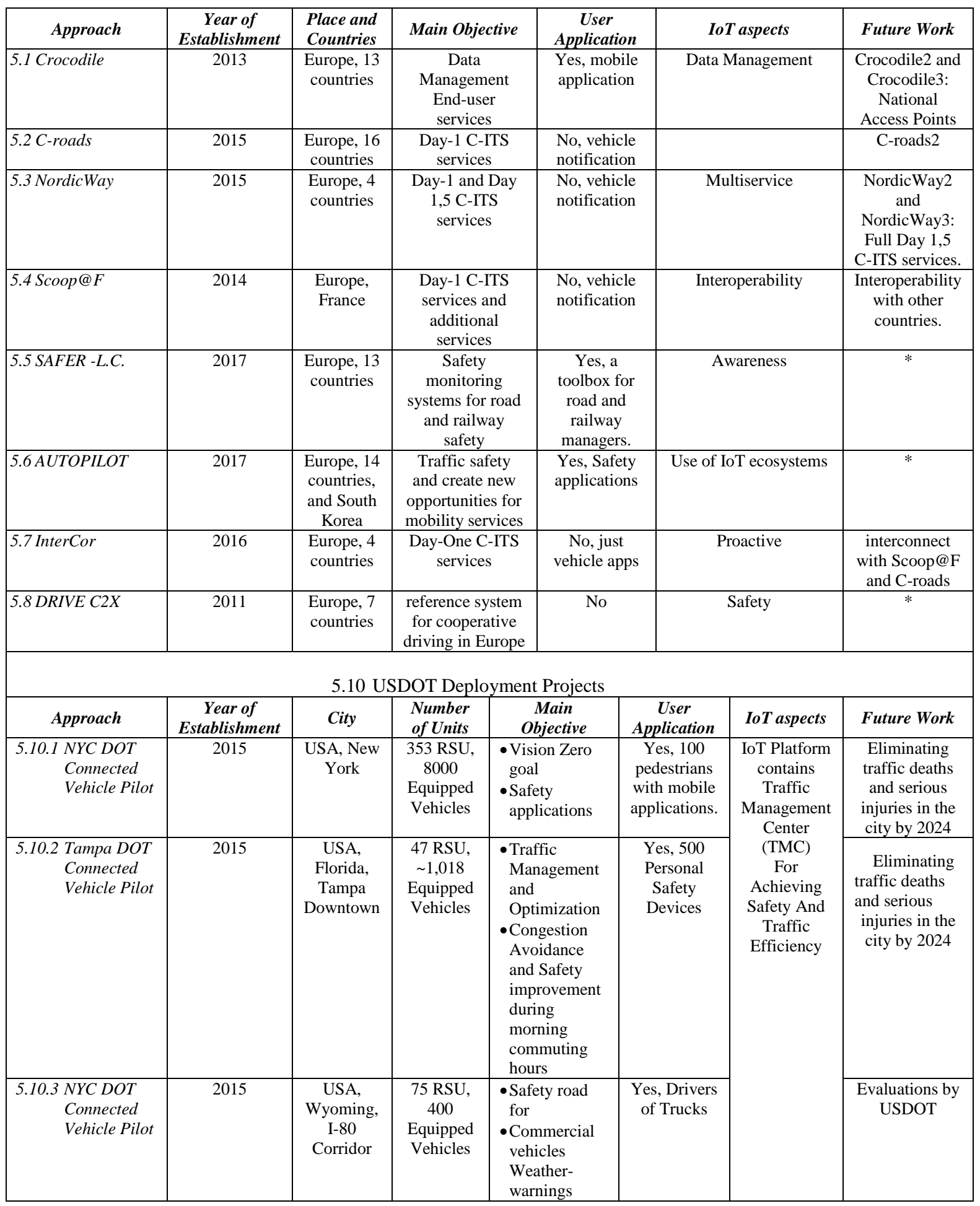

to tackle several research challenges regarding multimodal mobility, the hybrid/heterogeneous nature of V2X communication technologies, and many other factors.

The mixing of $\mathrm{V} 2 \mathrm{X}$ with IoT is a natural evolution step represented in smart mobility applications such as vehicle monitoring and autonomous vehicles, building smart cities such as public warning systems in critical infrastructures to manage different situations, creating smart energy such as future energy grids to charge cars, involving in intelligent manufacturing such as high-performance production comes for collected data and alerting service from intelligent vehicles. In addition to enhancing innovative electronic health use-cases, implementing advanced sensors infrastructure and emergency response systems in cars will also help drive safety. A whole new set of services and 
applications could arise. Our survey concludes that any C-ITS project relates to IoT aspects which will add huge benefits and privileges to be reliable and operational services.

\section{AUTHOR CONTRIBUTIONS}

H. Hejazi: Conceptualization, Theoretical analysis, Writing.

L. Bokor: Supervision, Review and editing.

\section{REFERENCES}

[1] D. Evans, How the Next Evolution of the Internet Is Changing Everything, Cisco Internet Business Solutions Group (IBSG), (2011). http://115.112.165.74:81/Krishna

Akalamkam/digital marketing/articles/The Internet of Things_.pdf

[2] B. Umar, H. Hejazi et al, Evaluation of IoT Device Management Tools, ACCSE 2018: The Third International Conference on Advances in Computation, Communications and Services, (2018), pp. 22-26.

[3] A. Napolitano, Implementation of a MECbased vulnerable road user warning system, Jul. 2019, pp. 1-6.

https://doi.org/10.23919/EETA.2019.8804497

[4] E. Torres-Zapata, V. Guerra et al, Vehicular communications in tunnels using VLC, Jul.2019, pp. 1-6.

https://doi.org/10.1109/ConTEL.2019.884850 $\underline{0}$

[5] A. Mai and D. Schlesinger, A Business Case for Connecting Vehicles, April, (2011). https://www.cisco.com/c/dam/en_us/about/ac7 9/docs/mfg/ConnectedVehicles_Exec_Summary.pdf

[6] S. GSMA, GSMA Connected Living programme: mAutomotive Executive summary, (2012).

https://www.gsma.com/iot/wp-

content/uploads/2012/04/gsmaconnectingcarst hetechnologyroadmapv2.pdf

[7] SBD, Connected Car Global Forecast 2015, (2015) [cited 2020-06-19].

https://www.sbdautomotive.com/files/sbd/pdfs 1536\%20connected\%20car\%20forecast $\% 20 \mathrm{ib}$ $\% 2015 . \mathrm{pdf}$

[8] Pwc, Connected car report 2016, PwC 2016, (2016) [cited 2020-06-19]. www.pwc.com/digital

[9] I. Ivanov, C. Maple et al, Cyber security standards and issues in V2X communications for Internet of vehicles, IET Conference Publications, 2018, vol. 2018, no. CP740, pp. $1-6$.

https://doi.org/10.1049/cp.2018.0046

\section{DISCLOSURE STATEMENT}

The authors declare that they have no known competing financial interests or personal relationships that could have appeared to influence the work reported in this paper.

\section{ORCID}

H. Hejazi https://orcid.org/0000-0002-9478-4197

L. Bokor https://orcid.org/0000-0003-1870-8544

[10] W. Anwar, N. Franchi et al, Physical layer evaluation of V2X communications technologies: 5G NR-V2X, LTE-V2X, IEEE 802.11bd, and IEEE 802.11p, IEEE Vehicular Technology Conference, 2019, vol. 2019Septe, pp. 1-7. https://doi.org/10.1109/VTCFall.2019.889131 $\underline{3}$

[11] S. Datta, J. Harri et al, Integrating connected vehicles in Internet of Things ecosystems: Challenges and solutions, WoWMoM 2016 17th International Symposium on a World of Wireless, Mobile and Multimedia Networks, (2016), pp. 1-6. https://doi.org/10.1109/WoWMoM.2016.7523 574

[12] K. Kiela, M. Jurgo et al, Structure of V2X-IoT framework for ITS applications, 2020 43rd International Conference on Telecommunications and Signal Processing, TSP 2020, 2020, pp. 229-234. https://doi.org/10.1109/TSP49548.2020.91635 $\underline{39}$

[13] A. A. Brincat, F. Pacifici et al, The Internet of Things for Intelligent Transportation Systems in Real Smart Cities Scenarios, IEEE 5th World Forum on Internet of Things, WF-IoT 2019 Conference Proceedings, 2019, pp. 128-132. https://doi.org/10.1109/WF-IoT.2019.8767247

[14] S. Husain, A. Kunz et al, An overview of standardization efforts for enabling vehicularTo-everything services, 2017 IEEE Conference on Standards for Communications and Networking, CSCN 2017, Oct. 2017, pp. 109114. https://doi.org/10.1109/CSCN.2017.8088607

[15] R. Chitanvis, N. Ravi et al, Collision avoidance and Drone surveillance using Thread protocol in V2V and V2I communications, Proceedings of the IEEE National Aerospace Electronics Conference, NAECON, Jul. 2019, vol. 2019July, pp. 406-411. https://doi.org/10.1109/NAECON46414.2019. $\underline{9058170}$ 
[16] J. Lozano and T. Mateo, Review on V2X, I2X, and P2X Communications and Their Applications: A Comprehensive Analysis over Time, Sensors, 2019, vol. 19, no. 12, p. 2756. https://doi.org/10.3390/s19122756

[17] J. Choi, V. Marojevic, and C. DIetrich, Measurements and Analysis of DSRC for V2T Safety-Critical Communications, IEEE Vehicular Technology Conference, 2018, vol. 2018-August, pp. 1-5. https://doi.org/10.1109/VTCFall.2018.869103 $\underline{2}$

[18] R. Jurgen, V2V/V2I Communications for Improved Road Safety and Efficiency, V2V/V2I Communications for Improved Road Safety and Efficiency, SAE, 2012, pp.i-viii.

[19] U.S. Department of Transportation, V2P Devices and Applications, p. 2004, (2005), [cited 2020-06-21]. www.its.dot.gov

[20] WHO, Global Status Report on Road Safety 2018, (2018).

https://www.who.int/publications/i/item/97892 41565684

[21] A. Hussein, F. García et al, P2V and V2P communication for pedestrian warning on the basis of autonomous vehicles, IEEE Conference on Intelligent Transportation Systems, Proceedings, ITSC, Dec. 2016, pp. 2034-2039. https://doi.org/10.1109/ITSC.2016.7795885

[22] K. Lakhtaria, Analyzing Zone Routing Protocol in MANET Applying Authentic Parameter, 2010, pp. 114-118. http://arxiv.org/abs/1012.2510

[23] F. Barsotti, A. Caruso, and S. Chessa, The localized vehicular multicast middleware: A framework for Ad Hoc inter-vehicles multicast communications, WSEAS Transactions on Communications, 2006, vol. 5, no. 9, pp. 17631768.

[24] C. Sharma and N. Gondhi, Communication Protocol Stack for Constrained IoT Systems, 2018 3rd International Conference On Internet of Things: Smart Innovation and Usages (IoTSIU), 2018, pp. 1-6.

https://doi.org/10.1109/IoTSIU.2018.8519904

[25] H. Hejazi, Investigation and Testing IoT Platforms Manufacture and Architecture, Master thesis, Budapest University of Technology and Economics (2018). https://diplomaterv.vik.bme.hu/en/Theses/Inve stigation-and-Testing-IoT-Platforms

[26] C. Liu, K. Chau et al, Opportunities and challenges of vehicle-to-home, vehicle-tovehicle, and vehicle-to-grid technologies, Proceedings of the IEEE, 2013, vol. 101, no. 11, pp. 2409-2427, https://doi.org/10.1109/JPROC.2013.2271951
[27] V. Monteiro, J. Pinto, J. Afonso, Operation Modes for the Electric Vehicle in Smart Grids and Smart Homes: Present and Proposed Modes, IEEE Transactions on Vehicular Technology, 2016, vol. 65, no. 3, pp. 10071020. https://doi.org/10.1109/TVT.2015.2481005

[28] A. Bustamante, S. Inca et al, Design of a V2V communications antenna based on LTE technology and IEEE802.11p standard, Proceedings of the 2017 IEEE 24th International Congress on Electronics, Electrical Engineering and Computing, INTERCON 2017, Aug. 2017, pp. 1-4. https://doi.org/10.1109/INTERCON.2017.807 9710

[29] R. Weber, J. Misener, V. Park, C-V2X - A communication technology for cooperative, connected and automated mobility, 24. ITGSymposium on Mobile Communication Technologies and Applications, 2020, pp. 111116.

[30] H. Abou-Zeid, F. Pervez et al, Cellular V2X Transmission for Connected and Autonomous Vehicles Standardization, Applications, and Enabling Technologies, IEEE Consumer Electronics Magazine, 2019, vol. 8, no. 6, pp. 91-98.

https://doi.org/10.1109/MCE.2019.2941467

[31] K. Ahmed, M. Lee, Secure LTE-Based V2X Service," IEEE Internet of Things Journal, 2018, vol. 5, no. 5, pp. 3724-3732. https://doi.org/10.1109/JIOT.2017.2697949

[32] H. Hejazi, H. Rajab et al, survey of platforms for massive IoT, 2018 IEEE International Conference on Future IoT Technologies, Future IoT 2018, Mar. 2018, vol. 2018January, pp. 1-8. https://doi.org/10.1109/FIOT.2018.8325598

[33] A. Kunz, L. Nkenyereye, J. Song, 5G Evolution of Cellular IoT for V2X, 2018 IEEE Conference on Standards for Communications and Networking (CSCN), 2018, pp. 1-6. https://doi.org/10.1109/CSCN.2018.8581830

[34] C. Storck, F. Duarte-Figueiredo, A 5G V2X ecosystem providing Internet of vehicles," Sensors (Switzerland), 2019, vol. 19, no. 3, p. 550 . https://doi.org/10.3390/s19030550

[35] M. Agiwal, A. Roy, N. Saxena, Next generation $5 \mathrm{G}$ wireless networks: A comprehensive survey, IEEE Communications Surveys and Tutorials, 2016, vol. 18, no. 3. Institute of Electrical and Electronics Engineers Inc., pp. 1617-1655. https://doi.org/10.1109/COMST.2016.253245 $\underline{8}$

[36] $\underline{S}$. Singh, D2D and V2X Communications, 5G System Design, John Wiley \& Sons, Ltd, 2018, pp. 409-449. 
https://doi.org/10.1002/9781119425144.ch14

[37] B. Gopal, P. Kuppusamy, A Comparative Study on $4 \mathrm{G}$ and $5 \mathrm{G}$ Technology for Wireless Applications, IOSR Journal of Electronics and Communication Engineering, 2015, vol. 10, no. 6, pp. 2278-2834.

[38] S. Chen, Vehicle-to-Everything (v2x) Services Supported by LTE-Based Systems and $5 \mathrm{G}$, IEEE Communications Standards Magazine, 2017, vol. 1, no. 2, pp. 70-76. https://doi.org/10.1109/MCOMSTD.2017.170 $\underline{0015}$

[39] P. Gaudillat, I. Antonopoulos et al, Best Environmental Management Practice for the Public Administration Sector. 2017. https://doi.org/10.2760/202143

[40] E. Strickland, 3 Ways Ford Cars Could Monitor Your Health - IEEE Spectrum, IEEE Spectrum. (2017) [cited 2021-04-05].

https://spectrum.ieee.org/the-humanos/biomedical/diagnostics/3-ways-ford-carscould-monitor-your-health

[41] W. Fehr, T. Lusco, et al, Southeast Michigan 2014 Test Bed project architecture update: Developing, refining and implementing the USDOT's Connected Vehicle Reference Implementation Architecture, 2014 International Conference on Connected Vehicles and Expo, ICCVE 2014 Proceedings, 2014, pp. 16-23. https://doi.org/10.1109/ICCVE.2014.7297535

[42] Crocodile, Harmonisation of Data Exchange, Results Of The Crocodile Project, 2016.

https://www.itsplatform.eu/filedepot_download/1838/5838

[43] Crocodile, IMPLEMENTATION OF HARMONISED INFORMATION EXCHANGE RESULTS OF THE CROCODILE 2 PROJECT, 2019.

https://www.itsplatform.eu/filedepot_download/1838/6552

[44] C-Roads, Core Members: C-Roads, (2019) [Cited 2020-02-02]. https://www.c-roads.eu/pilots/coremembers.html

[45] C. Platform, Specification for interoperability of backend hybrid C-ITS communication, 2019.

https://itscorridor.mett.nl/c-

its+corridor/Achtergronddocumenten/handlerd ownloadfiles.ashx?idnv=1514256

[46] A. Froetscher, B. Monschiebl, C-roads: Elements of C-ITS service evaluation to reach interoperability in Europe within a wide stakeholder network: Validation steps and comparative elements used in a living lab environment in Austria, IEEE Vehicular Technology Conference, Jul. 2018, vol. 2018June, pp. 1-5.
https://doi.org/10.1109/VTCSpring.2018.8417 874

[47] C-ROADS, C-ITS deployment takes off, increasing road safety and decreasing congestion, (2019) [cited 2021-02-19].

https://www.car-2-car.org/press-media/pressreleases/press-details/c-its-deployment-takesoff-increasing-road-safety-and-decreasingcongestion-80/

[48] Z. Lokaj, M. Srotyr et al, Technical part of evaluation solution for cooperative vehicles within C-ROADS CZ project, 2020 Smart City Symposium Prague (SCSP), 2020, pp. 1-5. https://doi.org/10.1109/SCSP49987.2020.9133 885

[49] NordicWay, Deployment Roadmap Recommendations for future work NordicWay, (2017). https://uploadsssl.webflow.com/5c487d8f7febe4125879c2d8/ 5c5c02c69c00c4851bedf1cb_NordicWay_Fin al\%20Report.pdf

[50] Nordicway2, (2017) [cited 2020-02-06]. https://www.nordicway.net/

[51] SCOOP@F Part 2, EUROPA - SCOOP@F Part 2 TRIMIS - European Commission, (2018) [cited 2020-02-06]. https://trimis.ec.europa.eu/project/scoopf-part$\underline{2}$

[52] D. Kountche, J. Bonnin, H. Labiod, The problem of privacy in cooperative intelligent transportation systems (C-ITS), 2017 IEEE Conference on Computer Communications Workshops, INFOCOM WKSHPS 2017, Nov. 2017, pp. 482-486.

https://doi.org/10.1109/INFCOMW.2017.8116 424

[53] SCOOP, SCOOP Cross Tests : a key step for European interoperability - SCOOP Project: connected road and vehicle (2019) [cited 202002-06].

http://www.scoop.developpementdurable.gouv.fr/en/scoop-cross-tests-a-keystep-for-european-a43.html

[54] A. Boniou, About SAFER-LC - SAFER-LC project, SAFER-LC project, (2020) [cited 2020-02-07]. https://safer-lc.eu/about-safer-lc

[55] SAFER-LC, SAFER Level Crossing By Integrating And Optimizing Road-Rail Infrastructure Management And Design, (2017) [cited 2020-02-07] www.safer-lc.eu

[56] European IoT Large-Scale Pilots, European IoT Large-Scale Pilots Programme Broachure, (2018).

https://european-iot-pilots.eu/wpcontent/uploads/2018/06/IOT-Booklet-Def.pdf 
[57] S. Antipolis, D. Brevi, R. Scopigno, "linksfoundation.com LINKS ACTIVITIES ON VULNERABLE ROAD USERS, (2019).

https://autopilot-project.eu/wpcontent/uploads/sites/3/2019/03/LINKSVRUs-activitiesv01.pdf

[58] G. Larini, Autonomous Driving Progressed by oneM2M: The Experience of the AUTOPILOT Project, 2019 European Conference on Networks and Communications, EuCNC 2019, Jun. 2019, pp. 204-208.

https://doi.org/10.1109/EuCNC.2019.8801948

[59] Intercor, Validation event InterCor in the Netherlands, pp. 4-5.

https://intercor-project.eu/wpcontent/uploads/sites/15/2017/10/Factsheet_In tercor-Testfest-DEF.pdf

[60] G. Crockford, B. Netten, P. Wadsworth, Establishing a common approach to evaluating the InterCor C-ITS pilot project, IEEE Vehicular Technology Conference, Jul. 2018, vol. 2018-June, pp. 1-2.

https://doi.org/10.1109/VTCSpring.2018.8417 $\underline{875}$

[61] Mayaivanova, DRIVE C2X 27/06/2014 Deliverable D55.1 Version 2.1 I Report on compliance of DRIVE C2X system and applications with international ITS standards 201 Deliverable D55.1 Report on compliance of DRIVE C2X system and applications with international ITS standard, (2014).

[62] Drive C2X, Drive C2X Project, p. 2, (2011) [cited 2021-02-21]. http://drive-c2x.eu/project

[63] Cooperative Connected and Automated Mobility (CCAM). MDPI, 2020. https://doi.org/10.3390/books978-3-03928159-6

[64] ECo-AT, European Corridor - Austrian Testbed for Cooperative Systems- Publication Event of Release 1 Documents, (2015). http://eco-at.info/downloads206.html?file=tl files/dynamic dropdown/upl oads/Presse/Praesentationen/ECoAT_Publication\%20Event\%20of\%20Release \%201\%20Documents 20150115.pdf

[65] Ejct, European Center for Information and Communication Technologies - ejct, (2013) [cited 2020-06-14]. https://www.eict.de/projekte/\#project-19

[66] C-ITS, Cooperative Intelligent Transport Systems and Services -Projects, (2019) [cited 2020-06-14]. https://www.car-2-car.org/about-c-its/

[67] K. Hartman, connected-vehicle-pilotdeployment-program, Program Evaluation Survey Plan,(2019). https://rosap.ntl.bts.gov/view/dot/42403/dot 4 2403 DS1.pdf
[68] C. Vargas, Together for Safer Roads, Anheuser-Busch First to Join NYC Connected Vehicle PilotNew Public-Private Partnership Supports City's Vision Zero Goals TogetherForSaferRoads, pp. 5-7, (2019).

[69] United States Department of Transportation, Intelligent Transportation Systems - Connected Vehicle Pilot Deployment Program, (2019) [cited 2020-02-03].

https://www.its.dot.gov/pilots/pilots_wydot.ht m

[70] DOT Connected Vehicle Pilot, THEA CONNECTED VEHICLE PILOT-FACT SHEET. (2019) [cited 2020-02-03]. https://www.its.dot.gov/pilots/pilots thea.htm

[71] THEA, Connected Vehicle Pilot, (2019) [cited 2021-04-01]. https://www.fdot.gov/traffic/its/projectsdeploy/cv/maplocations/thea-cvp.shtm

[72] ITS Joint Program Office, "WyomingCVPilot_Factsheet," vol. 80, (2016).

https://www.its.dot.gov/factsheets/pdf/Wyomi ngCVPilot_Factsheet.pdf

[73] DOT Connected Vehicle Pilot, Wyoming DOT Connected Vehicle Pilot Deployment Program, (2015) [cited 2021-04-01]. https://www.its.dot.gov/pilots/pilots_wydot.ht $\underline{\mathrm{m}}$

[74] THEA, Intelligent Transportation Systems ITS Deployments, (2019) [cited 2021-04-01]. https://www.its.dot.gov/pilots/cv_pilot_world wide.htm

[75] Austroads, Austroads Strategic Plan 20202024, AP-C29-20 (2020).

[76] P. van Dijk, S. Australia, Privacy Impact Assessment (PIA) for Cooperative Intelligent Transport System (C-ITS) data messages, (2017) [cited 2021-05-14]. www.austroads.com.au

[77] M. Fallgran, On Selected V2X Technology Components and Enablers from the 5GCAR Project, IEEE International Symposium on Broadband Multimedia Systems and Broadcasting, BMSB, 2018, pp. 1-5. https://doi.org/10.1109/BMSB.2018.8436731

[78] M. Fallgren, "White Paper 5GCAR : Executive Summary White Paper 5GCAR: Executive Summary," no. 761510, (2019).

https://5gcar.eu/wpcontent/uploads/2019/12/5GCAR-ExecutiveSummary-White-Paper.pdf

[79] G. Vazquez, Deliverable D5.1 Description of 5GCroCo Business Potentials Deliverable D5.1 Description of 5GCroCo Business Potentials Description of 5GCroCo Business Potentials, Marc, (2019) [cited 2021-05-14]. http://www.5g-ppp.eu

[80] F. Ertico, F. Fischer, J. Ferragut, 5G HarmoniseD Research and TrIals for serVice 
Evolution between EU and China D4 . 1 : V2X Development and Test Plan, (2019).

[81] HUAWEI, Wuxi Internet of Vehicle (C-V2X) Project Use Cases list - Huawei United Kingdom, www.huawei.com, (2019) [cited 2020-12-06].

https://www.huawei.com/en/industryinsights/outlook/mobile-broadband/lte/usecases/wuxi-internet-of-vehiclec-project-use$\underline{\text { cases }}$
[82] P. Lancia and J. Sinnott, AT, Ford, Nokia and Qualcomm Launch Cellular-V2X Connected Car Technology Trials Planned for the San Diego Regional Proving Ground with Support From McCain | Qualcomm, (2017) [cited 202012-06].

https://www.qualcomm.com/news/releases/20 17/10/31/att-ford-nokia-and-qualcommlaunch-cellular-v2x-connected-car-technology

This article is an open access article distributed under the terms and conditions of the Creative Commons Attribution NonCommercial ( $C C B Y-N C$ 4.0) license. 